\title{
The importance of level stratification for quantitative MR studies of lumbar intervertebral discs: a cross-sectional analysis in 101 healthy adults
}

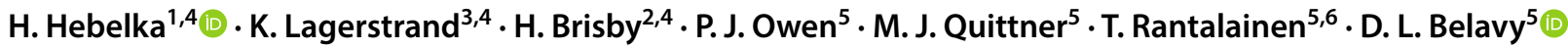

Received: 8 February 2019 / Revised: 31 May 2019 / Accepted: 1 July 2019 / Published online: 12 July 2019

(c) The Author(s) 2019

\begin{abstract}
Purpose To investigate whether quantitative T2-times depend on lumbar intervertebral disc (IVD) level.

Methods The lumbar spine (Th12/L1-L5/S1) of 101 participants $(53.5 \%$ female, $30.0[ \pm 3.6]$ years, $173.5[ \pm 9.6] \mathrm{cm}$ and $69.9[ \pm 13.4] \mathrm{kg}$ ), without history of back pain, was examined on a 3T scanner with sagittal T2-mapping. All IVDs were stratified according to Pfirrmann grade and lumbar level, with mean T2-time determined for the entire IVD volume and in five subregions of interests.

Results Significant level-dependent T2-time differences were detected, both for the entire IVD volume and its subregions. For the entire IVD volume, Pfirrmann grade 2 IVDs displayed 9-18\% higher T2-times in Th12/L1 IVDs compared to L2/ L3-L5/S1 IVDs $(0.001>p<0.004)$ and significantly different T2-times in L1/L2-L2/L3 IVDs compared to most of the IVDs in the lower lumbar spine. In Pfirrmann grades 1, 3 and 4 IVDs, no significant level-dependent T2-time differences were observed for the entire IVD. More pronounced results were observed when comparing IVD subregions, with significant level-dependent differences also within Pfirrmann grade 1 and grade 3 IVDs. For example, in posterior IVD subregions mean T2-time was 80-82\% higher in Th12/L1 compared to L3/L4-L4/L5 Pfirrmann grade 1 IVDs $(p<0.05)$ and 10-14\% higher in L5/S1 compared to L3/L4-L4/L5 Pfirrmann grade 3 IVDs $(0.02>p<0.001)$.

Discussion Significant level-dependent T2-time differences within several Pfirrmann grades, both for the entire IVD volume and for multiple IVD subregions, were shown in this large cohort study. The T2-time differences between levels existed in both non-degenerated and degenerated IVDs. These findings show the importance of stratifying for lumbar level when quantitative IVD studies are performed using T2-mapping.
\end{abstract}

\section{Graphic abstract}

These slides can be retrieved under Electronic Supplementary Material.
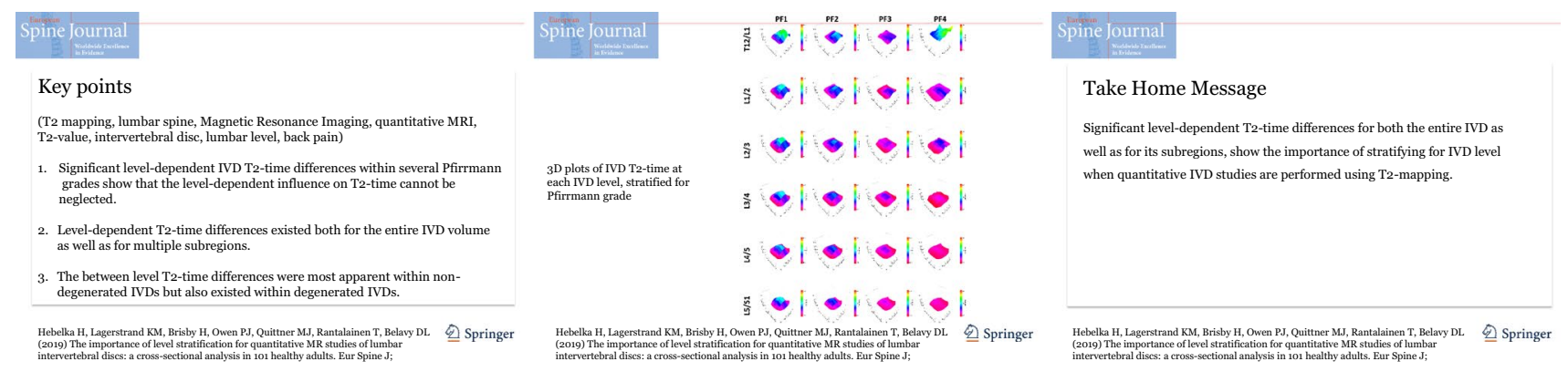

Keywords T2 mapping · Magnetic resonance imaging · Quantitative MRI $\cdot$ Intervertebral disc $\cdot$ Lumbar level

Electronic supplementary material The online version of this article (https://doi.org/10.1007/s00586-019-06059-1) contains supplementary material, which is available to authorized users.

Extended author information available on the last page of the article 


\section{Introduction}

Despite advancement of quantitative magnetic resonance imaging (MRI) techniques within the spinal field, MRI of the spine is still conduced with traditional T1- and $\mathrm{T} 2$-weighted sequences in the clinic. There are limitations with grading systems based on these methods. For example, the commonly used Pfirrmann grading is a subjective classification system of intervertebral disc (IVD) degeneration based on these methods, where objective, detailed tissue characterization is not allowed [1]. Further, traditional MRI provides only a static image of the spine in a relaxed supine position without any information regarding mechanical impact on the spinal structures. In order to advance within IVD diagnostics, both for the search of biomarkers of pain and for determining the impact of interventions (e.g. stem cell therapies, surgical outcomes, non-invasive therapies) and spinal biomechanics, detailed and robust methods for assessing quantitative and functional IVD characteristics are necessary.

Quantitative MRI techniques, such as T2-mapping, show promise to reflect both structural and functional IVD characteristics non-invasively [2-5]. It has been established that T2-relaxation times provide continuous and objective grading of degeneration, with reduced T2-relaxation times correlating well with increased grade of degeneration [6-8]. Recent studies, including studies imaging the spine in loaded position, indicate regional IVD differences when comparing T2-maps between low back pain (LBP) patients and controls [9-12], which is promising in the search for pain predictors.

Numerous studies regarding quantitative MRI and lumbar IVD data exist; however, the majority are based on small cohorts and include divergent methodology, such as different IVD segmentation methods and the use of either axial or sagittal sequencing [3, 4, 6, 8-10,13-18], thus limiting comparison between studies. Further, variation between different motion segments can be expected due to natural IVD size and loading angles [19]. It is known that IVD composition, as well as its ability to resist and respond to load, differs depending on IVD segments in the lumbar spine $[11,20,21]$. Despite this, no study has, in a standardized manner in a large cohort, elucidated whether and how quantitative MRI data vary depending on at what lumbar level the IVD is located, neither in an asymptomatic nor in a symptomatic cohort. Some studies have reported level-dependent $\mathrm{T} 2$-time differences, including studies investigating the impact of load on T2-time [2-4, 11]. However, these studies have been based on small cohorts, so their results should be interpreted with caution. Since several quantitative MRI studies have reported differences within posterior IVD regions between LBP patients and controls [9-12], it seems important to elucidate how quantitative MRI data depend on IVD level, regarding not only the entire IVD but also potential impact on its subregions.

As a base for future research within the IVD field, the aim of this study was to investigate whether quantitative IVD T2-times depend on lumbar level in a large population with no prior history of spinal pain.

\section{Methods}

\section{Participants}

The lumbar spine of 101 volunteers, without prior history of back pain, was examined. This exploratory study was conducted as a secondary analysis of a pre-existing data set from a cross-sectional project examining the effect of physical activity and inactivity on the lumbar spine in 101 healthy individuals aged $25-35$ years [5, 22]. Exclusion criteria included current spinal pain, history of spinal surgery, history of traumatic injury to the spine, known scoliosis for which prior medical consultation has been sought, current or prior smoker, known claustrophobia and possible pregnancy.

\section{MRI}

Participants sat for $>20$ min prior to entering the MR scanner. The entire spinal volume from the T11 vertebral body to the sacrum was examined in all subjects using a 3T Philips Ingenia scanner (Amsterdam, Netherlands). Sagittal T2-mapping was performed using spin-echo multi-echo sequences with eight echo times $(15.75,36.75,57.75,78.75$, $99.75,120.75,141.75$ and $162.75 \mathrm{~ms}$ ) from 12 sagittal anatomical slices (thickness $3 \mathrm{~mm}$; interslice distance $1.5 \mathrm{~mm}$; repetition time $2000 \mathrm{~ms}$, field of view $281 \times 281 \mathrm{~mm}$, image resolution $0.366 \mathrm{~mm}$ per pixel). For Pfirrmann grading, 15 T2-weighted sagittal images (thickness $3 \mathrm{~mm}$; interslice distance $1.5 \mathrm{~mm}$; repetition time $2600 \mathrm{~ms}$, echo time $70 \mathrm{~ms}$ ) encompassing the entire spine from left to right were collected.

IVDs from T12/L1 to L5/S1 were included in the analysis, with supernumerary lumbar IVDs (L6/S1 discs in eight participants) excluded from the analysis. One experienced radiologist graded all IVDs according to the Pfirrmann classification [1] on the T2-weighted images. IVD segmentation was performed manually on the T2-mapping sequence using the native "polygon selections" tool in the off-line software ImageJ (http://rsb.info.nih.gov/ij/). Each IVD was segmented on each image. A custom-written plugin ("ROI Analyzer";https://github.com/tjrantal/RoiAn alyzer https://sites.google.com/site/daniellbelavy/home/ roianalyser) then horizontally aligned the segmented IVD 
and calculated the signal intensity in each echo image in the entire IVD as well as in five regions of interests (ROI), from ROI1 anteriorly to ROI5 posteriorly (Fig. 1). The T2-time in each region was then calculated. IVDs were stratified on both Pfirrmann grade and lumbar level.

\section{Statistical analyses}

For each intervertebral level, $t$-tests were performed to compare the T2-time for the entire IVD between levels for each Pfirrmann grade. A similar analysis was performed for each individual IVD subregion. To minimize the risk of type I errors and aid interpretation of the findings, all $p$-values were adjusted by the false discovery rate method [23]. An alpha level of 0.05 was taken for statistical significance. The " $R$ " statistical environment (version 3.4.2, www.r-project.org) was used for all analyses. All values are reported as mean (SD), unless otherwise specified. When specifying between-level T2-time differences, values are given as per cent increase relative to the lower T2-time.

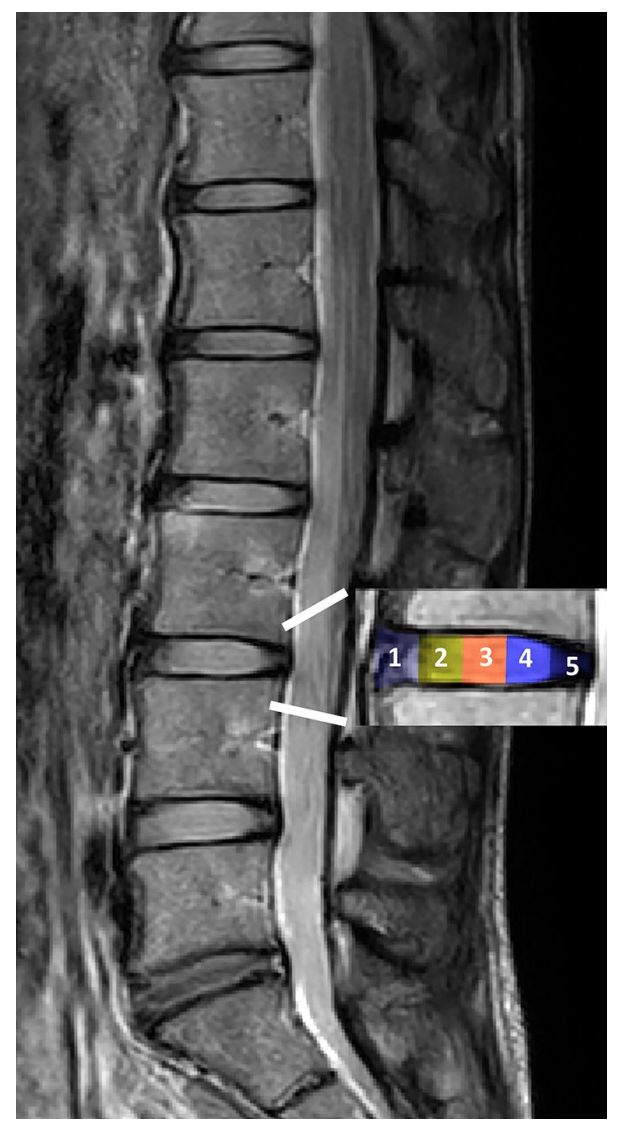

Fig. 1 Example of MR image with segmentation at the L3/L4 intervertebral disc level. Inset shows location of the subregions (ROI1-5) from anterior to posterior

\section{Results}

The 101 volunteers consisted of 47 men and 54 women of $30.0( \pm 3.6)$ years of age, $173.5( \pm 9.6) \mathrm{cm}$ height and weight of $69.9( \pm 13.4) \mathrm{kg}$. Significant level-dependent T2-time differences were detected for both the entire IVD volume and IVD subregions (Table 1; Fig. 2). The IVD distribution within each Pfirrmann grade at each lumbar level is displayed in Table 1.

\section{Entire IVD}

When stratifying the mean T2-times for the entire IVD according to Pfirrmann, level-dependent differences were detected in grade 2 IVDs, with a higher T2-time in Th12/ L1 IVDs (range 9-18\%) compared to all other lumbar levels except L1/L2 (0.001 $>p<0.004$; Table 2$)$ and significantly different T2-times in L1/L2-L2/L3 IVDs compared to most of the IVDs in the lower lumbar spine. For example, L1/L2 displayed 12\% higher mean T2 compared to L4/L5 IVDs $(p<0.001)$. In Pfirrmann grades 1,3 and 4 IVDs, no significant level-dependent T2-time differences were observed for the entire IVD.

\section{Subregions}

For Pfirrmann grade 1, level-dependent differences were noted in ROI4-5 (Table 1). Mean T2-time in ROI4 was 23\% higher in L2/L3 IVDs compared to L4/L5 IVDs ( $p=0.049)$, and in ROI5 Th12/L1 displayed a higher mean T2 (80-82\%) compared to L3/L4 and L4/L5 levels ( $p<0.05)$ (Table 2).

For Pfirrmann grade 2 IVDs, level-dependent T2-time differences were found between most lumbar levels regarding ROI4-5 and between Th12/L1 and several other levels regarding ROI3. For example, T2-time in ROI4-5 at Th12/ L1 IVDs was $29-40 \%$ higher compared to L5/S1 $(p<0.001)$. Not only IVDs distant to each other differed significantly in T2-time, but also IVDs adjacent to each other differed significantly in mean T2-time. For example, 5-7\% higher T2-time was found in L5/S1 IVDs compared to L4/L5 regarding ROI1-2 $(p<0.05)$ and in ROI3 a 7\% higher T2-time in L2/ L3 compared to L4/L5 ( $p=0.021)$ (Table 1).

In degenerated IVDs, i.e. Pfirrmann grade 3, leveldependent T2-time differences were detected in ROI5. For example, mean T2-time in ROI5 at L5/S1 was $14 \%$ $(p=0.001)$, respectively, $10 \%(p=0.013)$ higher compared to L3/L4 and L4/L5 IVDs (Table 2). Further, mean T2 in ROI1 at L1/L2 IVDs differed significantly to corresponding ROI at all lumbar levels except Th12/L1. For 
Table 1 The IVD T2-time distribution within each Pfirrmann grade at each lumbar level

\begin{tabular}{|c|c|c|c|c|c|c|}
\hline \multirow[t]{2}{*}{ Disc region $/ N$} & \multicolumn{6}{|l|}{ Lumbar level } \\
\hline & Th12/L1 & $\mathrm{L} 1 / \mathrm{L} 2$ & L2/L3 & $\mathrm{L} 3 / \mathrm{L} 4$ & L4/L5 & $\mathrm{L} 5 / \mathrm{S} 1$ \\
\hline & \multicolumn{6}{|l|}{ Pfirrmann grade 1} \\
\hline$N$ & 5 & 3 & 9 & 16 & 6 & 8 \\
\hline Entire IVD & $139.2(27.9)$ & $117.0(12.7)$ & $110.5(15.4)$ & $102.0(10.7)$ & $98.7(5.4)$ & $107.8(12.7)$ \\
\hline 1 & $103.6(16.0)$ & $89.5(4.3)$ & $89.3(14.1)$ & $84.1(8.6)$ & $88.9(6.3)$ & $94.9(15.4)$ \\
\hline 2 & $130.3(24.2)$ & $111.0(14.1)$ & $103.7(13.1)$ & $100.5(9.7)$ & $99.7(9.2)$ & $112.7(12.6)$ \\
\hline 3 & $165.8(39.2)$ & $138.5(18.0)$ & 133.2(20.9) & $120.5(16.5)$ & $112.3(8.3)$ & $121.7(17.5)$ \\
\hline 4 & $167.8(55.1)$ & $131.9(28.9)$ & $120.6(20.8) \mathrm{d}$ & $106.8(15.4)$ & $98.1(9.5) b$ & $104.6(15.7)$ \\
\hline \multirow[t]{2}{*}{5} & 139.1(32.6)cd & $107.0(14.7)$ & $88.3(13.6)$ & $76.4(7.4) \mathrm{f}$ & $77.1(3.5) \mathrm{f}$ & $85.3(10.5)$ \\
\hline & \multicolumn{6}{|l|}{ Pfirrmann grade 2} \\
\hline$N$ & 92 & 91 & 84 & 74 & 63 & 53 \\
\hline Entire IVD & 110.7(22.8)BCDE & 104.7(15.6)CDe & 101.4(11.6)CDF & 96.1(9.6)ABF & 93.8(9.4)ABF & 98.4(12.9)aF \\
\hline 1 & $91.0(16.5) \mathrm{d}$ & $87.9(11.5)$ & $92.5(20.8) \mathrm{cd}$ & $86.2(10.5) b$ & 85.7(8.5)bef & $90.4(10.3) \mathrm{d}$ \\
\hline 2 & $99.0(21.8)$ & $93.8(16.1) \mathrm{e}$ & $93.0(12.8) \mathrm{E}$ & $93.4(11.5) \mathrm{E}$ & $95.1(12.7) \mathrm{e}$ & 102.1(15.4)aBCd \\
\hline 3 & 121.1(29.4)CDE & 114.3(19.6)D & $112.8(16.7) \mathrm{d}$ & 109.9(14.1)F & 105.9(13.2)AbF & $108.3(19.0) \mathrm{F}$ \\
\hline 4 & 129.4(36.1)BCDE & 119.9(24.7)BCDE & 109.7(17.3)CDEF & $98.4(14.7) \mathrm{dF}$ & 92.2(11.7)ABcF & $92.6(14.3) \mathrm{ABF}$ \\
\hline \multirow[t]{2}{*}{5} & 109.6(31.0)ABCDE & 96.5(19.0)BCDEF & 85.8(11.2)CDF & 74.7(8.4)EF & 74.3(7.5)EF & 84.7(8.9)ABDF \\
\hline & \multicolumn{6}{|l|}{ Pfirrmann grade 3} \\
\hline$N$ & 3 & 4 & 7 & 9 & 16 & 21 \\
\hline Entire IVD & $102.3(15.0)$ & $84.2(8.4)$ & $86.1(9.3)$ & $87.3(9.2)$ & $85.5(7.1)$ & $85.1(5.4)$ \\
\hline 1 & $93.1(6.2)$ & 74.6(4.0)bcDE & $90.4(10.3) \mathrm{a}$ & $85.7(9.0) \mathrm{a}$ & 87.3(6.7)A & 88.5(8.9)A \\
\hline 2 & $97.7(19.2)$ & $75.1(10.1)$ & $81.0(12.9)$ & $87.6(10.4)$ & $86.9(9.1)$ & $86.0(8.7)$ \\
\hline 3 & $112.4(23.1)$ & $85.8(9.9)$ & $91.1(13.5)$ & $94.7(14.1)$ & $90.3(10.5)$ & $86.3(6.7)$ \\
\hline 4 & $108.3(12.3)$ & $96.1(15.3)$ & $85.8(9.5)$ & $83.5(11.3)$ & $81.0(7.6)$ & $80.2(6.6)$ \\
\hline \multirow[t]{2}{*}{5} & $97.5(8.3)$ & 84.8(3.5)bCD & $75.8(3.9) \mathrm{a}$ & 72.3(3.5)AE & 74.4(5.4)Ae & $82.2(9.3) \mathrm{Cd}$ \\
\hline & \multicolumn{6}{|l|}{ Pfirrmann grade 4} \\
\hline$N$ & 1 & 3 & 1 & 2 & 16 & 19 \\
\hline Entire IVD & $167.6(-)$ & $94.7(18.0)$ & $90.8(-)$ & $68.7(3.7)$ & $78.7(7.7)$ & $80.8(11.3)$ \\
\hline 1 & $168.5(-)$ & $90.8(15.1)$ & 81.1(-) & $75.1(3.0)$ & $86.7(7.5)$ & $84.1(10.2)$ \\
\hline 2 & $148.8(-)$ & $91.2(15.5)$ & $91.2(-)$ & $62.1(0.7)$ & $77.9(9.2)$ & $82.0(15.6)$ \\
\hline 3 & $163.3(-)$ & $98.4(20.8)$ & $102.3(-)$ & $66.3(1.5)$ & $77.6(9.8)$ & $80.0(13.0)$ \\
\hline 4 & $178.0(-)$ & $98.2(24.5)$ & $92.2(-)$ & 71.1(7.9) & $73.5(9.3)$ & 76.4(10.3) \\
\hline 5 & $247.0(-)$ & $96.8(24.4)$ & $84.9(-)$ & $71.6(8.7)$ & $78.8(7.9)$ & $80.0(8.7)$ \\
\hline
\end{tabular}

Values are mean (SD) T2-time in ms averaged across the entire intervertebral disc volume and the subregions 1-5. a, b, c, d, e, f or A, B, C, D, E, F refer, respectively, to a $p<0.05$ or $p<0.01$ for the difference to the L1/L2, L2/L3, L3/L4, L4/L5, L5/S1 or T12/L1 vertebral levels (Table 2)

Pfirrmann grade 4 IVDs, no level-dependent differences were displayed (Tables 1,2).

\section{Discussion}

This cross-sectional T2-mapping study, investigating whether quantitative IVD data depend on lumbar level in a large asymptomatic cohort, found significant level-dependent T2-time differences for both the entire IVD and its subregions. These results show the importance of stratifying for IVD level when quantitative IVD studies are performed using T2-mapping.
Significant differences in absolute T2-times between IVD levels are likely a reflection of the underlying physiological properties of the IVDs, where several factors might impact, such as tissue composition, relation to surrounding anatomical structures and loading angle. More specifically, it is known that the IVD composition and its ability to resist and respond to load vary between lumbar levels [20, 21], supporting our observations in the current study. In addition, substantial effect of lumbar level has been shown for several biomechanical metrics. The lumbosacral IVD is stiffer compared with the upper and mid-lumbar IVDs regarding axial rotation and lateral bending, and the viscoelastic IVD properties also depend on whether it belongs to the upper 
PF1

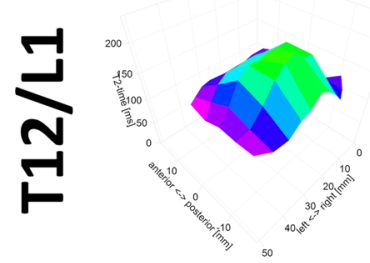

(1)
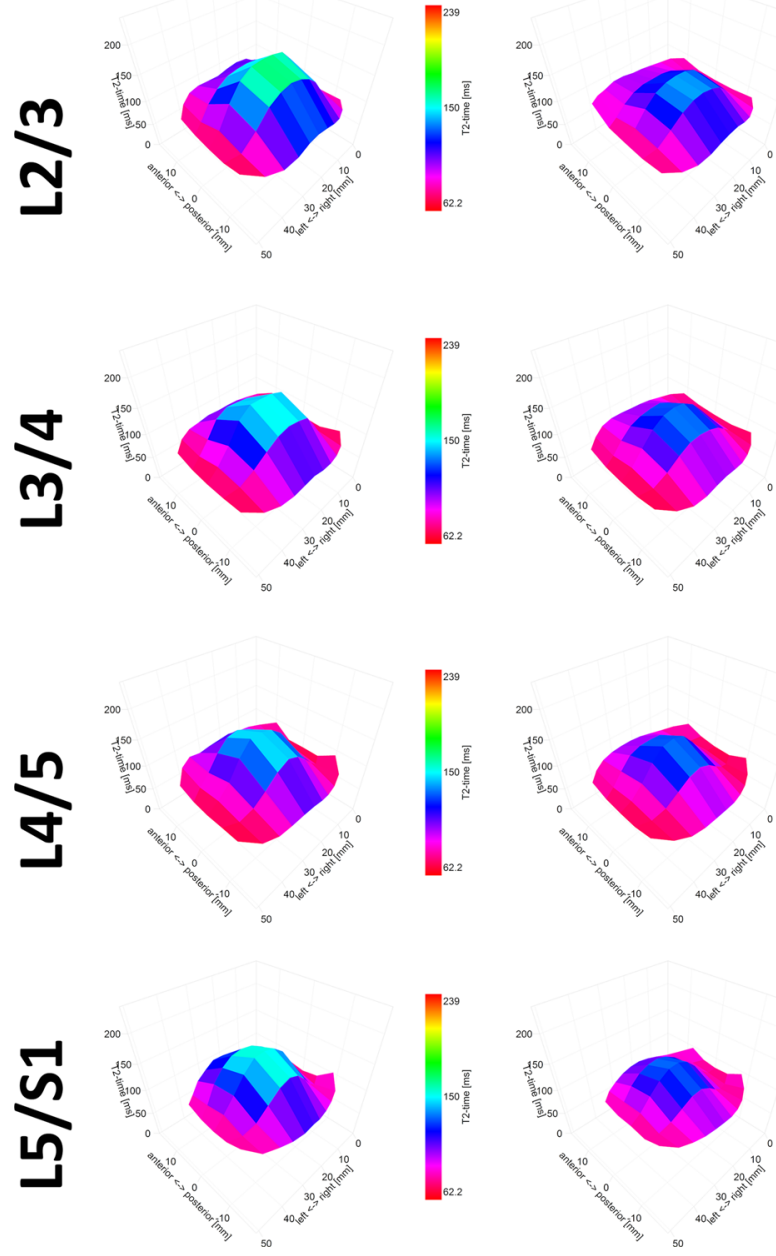

Fig. 2 3D plots of mean T2-time across the entire IVD volume. 3D plots illustrating the mean T2-time distribution across the entire IVD within each Pfirrmann grade at each lumbar level. The mean T2-time
PF3

239

$\mathrm{H}$

PF4

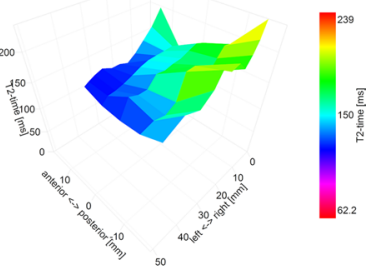

152.2
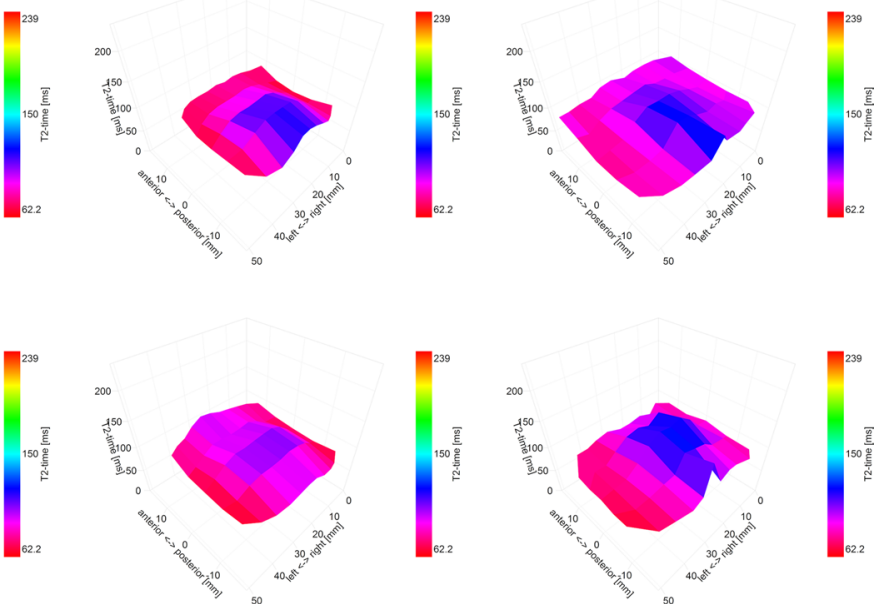

$\mathrm{i}$

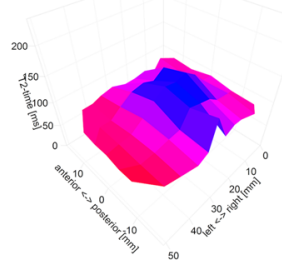

$\underbrace{239}_{62.2}$

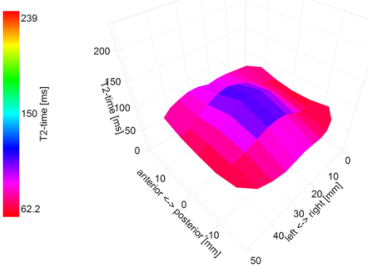

${ }_{02.2}^{239}$
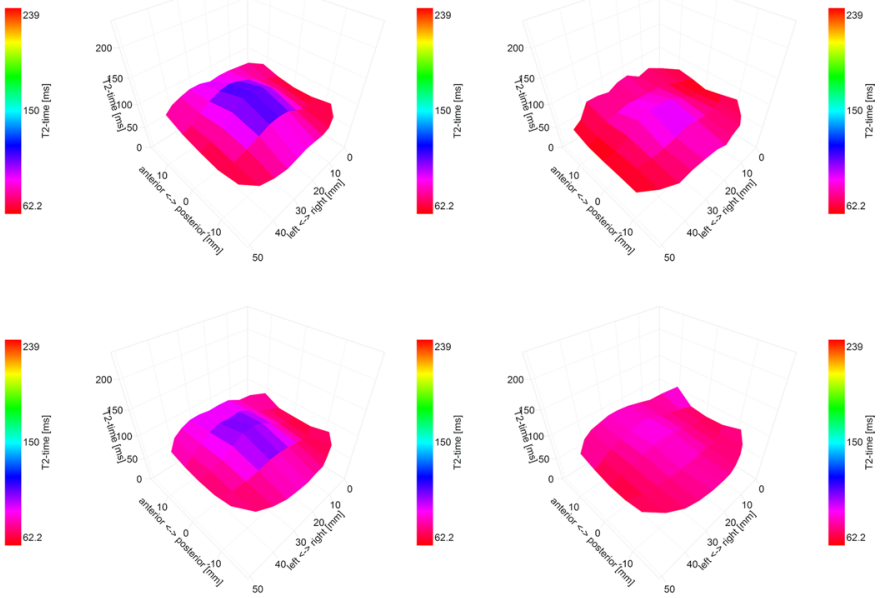

$\underbrace{2029}_{0.22}$
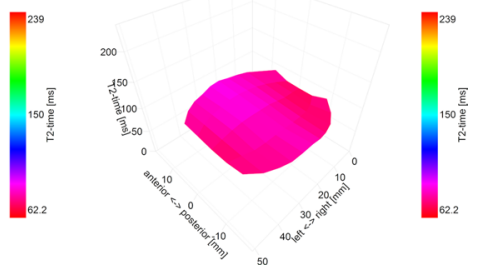

is displayed on the $y$-axis, and distribution across the IVD in anterior-posterior direction, respectively, from left to right is displayed on the $x$-axis and $z$-axis. PF Pfirrmann grade 


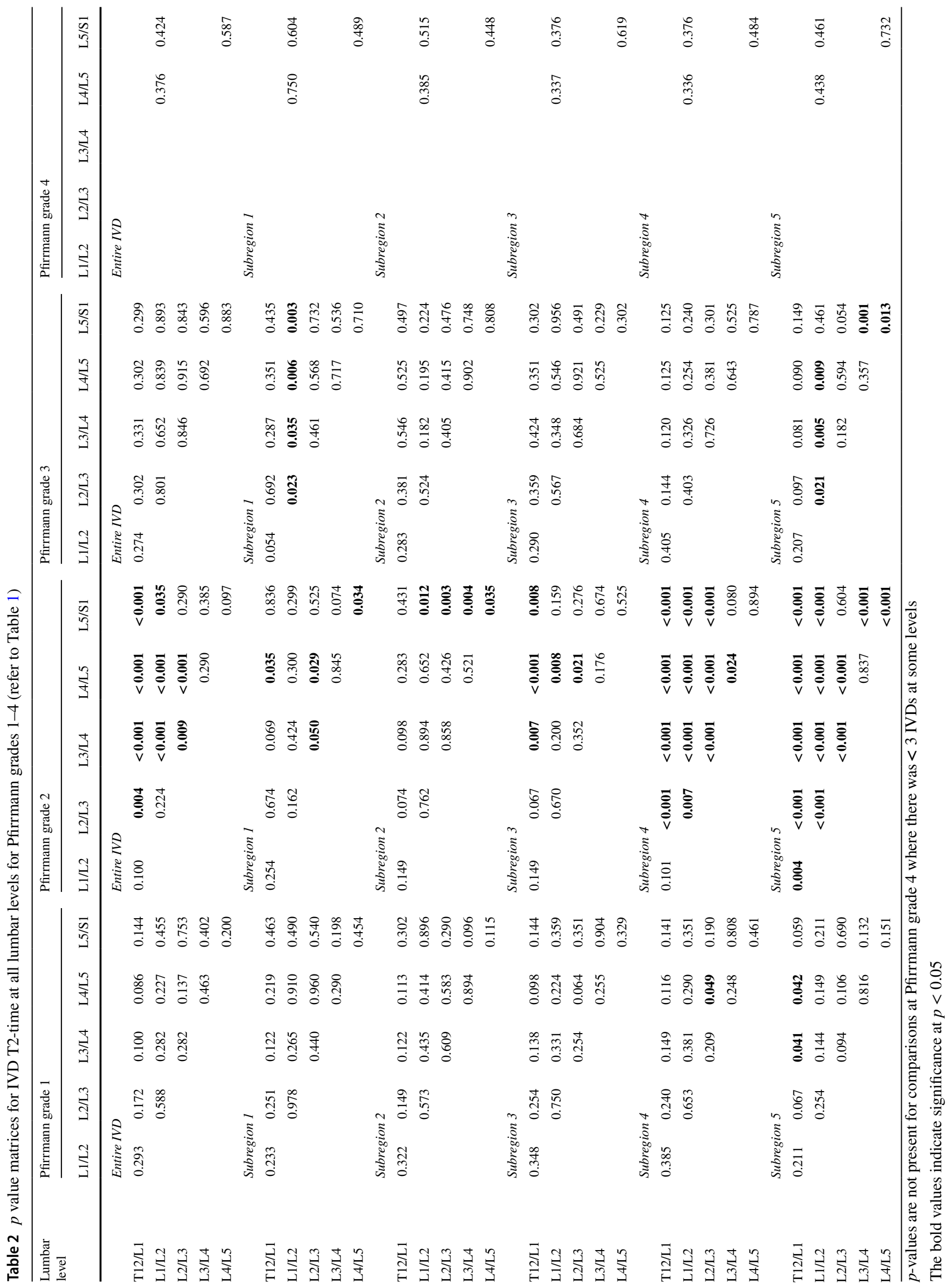


lumbar spine versus mid-lumbar or lumbosacral spine [20]. Further, Alexander et al. reported that the nucleus pulposus of L4/L5 and L5/S1 IVDs were the ones most affected in terms of sagittal migration, due to altered position, compared to lack of significant migration in upper lumbar spine [24]. Thus, it is not surprising that these structural and biomechanical differences between lumbar levels are reflected by level-dependent differences in T2-values.

In order to gain advanced knowledge regarding how degenerative IVD changes are related to LBP, the possibility to obtain both quantitative IVD measures, as well as detailed tissue characteristics, makes T2-mapping a viable option when studying the lumbar spine. Numerous T2-mapping studies of the IVD have been published in the last two decades [2, 4, 6, 7, 9-11, 13-15, 25]. However, the most existing quantitative MRI studies are based on small cohorts with divergent methodology, thus limiting comparison between studies. T2-mapping studies on lumbar IVDs of asymptomatic individuals have been conducted on cohorts ranging between 5 and 30 individuals [3, 4, 6, 7, 17, 18, 26], and similar studies on LBP patients are based on cohorts between 10 and 60 individuals [6, 8-11, 13-16, 25]. Hoppe et al. performed axial T2* mapping in 93 patients, but did not report any level specific data [15]. With few exceptions, level-dependent data are not reported in quantitative MRI studies of the IVDs. Furthermore, no prior study, to our knowledge, has published a large data set regarding T2-values stratified for lumbar level. Thus, the results of the current study fill the existing gap in the literature regarding how the lumbar level influences T2 data.

To combine quantitative MRI techniques with various positions and spinal loading has additionally shown promise to reflect functional characteristics of the spine, with concomitant level-dependent differences reported [2, 11, 27]. Nilsson et al. compared IVD T2 maps between conventional MRI and axial loading during MRI and found significantly induced change in L2/L3 and L5/S1, but not in the other lumbar IVDs, which may reflect the limited statistical power of their study, since the cohort consisted of only 11 patients. Further, Abdollah et al. [21] reported that the T2-weighted centre (mean position of the points in an ROI, weighted by their T2) was anteriorly displaced at L4/L5 but not at L5/ S1 during extension, also strengthening the argument for stratification for lumbar level when performing functional MRI studies of the lumbar spine.

Across all applicable Pfirrmann grades, the level-dependent T2-time differences were most persistent within posterior IVD parts, i.e. within ROI4-5, which represents the region between nucleus pulposus and annulus fibrosus, respectively, the posterior annulus. The finding of regional IVD differences in ROI4-5 between LBP patients and controls in several T2-mapping studies highlights the importance of subregional IVD analysis in the search of pain markers [9-12].
Adding the findings of the current study further shows the importance of not grouping all lumbar levels together when analysing quantitative IVD data. Considering the results in the current study, it cannot be excluded that the regional differences between patients and controls in previous quantitative MRI studies have been influenced by level-dependent differences. This notion calls for studies reproducing the regional differences between patients and controls also after stratifying for lumbar level since the confirmation of such findings might aid in the search for biomarkers of pain.

Our results with declining mean T2-times in caudal direction of the lumbar spine are consistent with the findings of Blumekratz et al. [6]. Also Chokan et al. and Yamabe et al. found differences in T2-times between lumbar levels, with, for example, lower T2-time in nucleus pulposus of L5/S1 as compared to L1/L2 [3, 4]. Differences in absolute values between these previous studies and our results are probably due to methodology inconsistencies, such as the use of axial T2-maps, lack of volumetric ROIs, higher mean age and the lack of adjustment for degeneration grade in previous studies.

Since degenerative changes are mostly common in the lower lumbar regions, not accounting for degeneration grade when analysing level-dependent T2-time influence seems doubtful. We speculate that the level-dependent differences in the current study could be due to the imperfections of the Pfirrmann grading scheme itself. However, even though such categorical qualitative classification systems are subjective and insensitive for within-grade inhomogeneities, they are still considered as reference classifications against which to compare new grading systems. We therefore chose to stratify for Pfirrmann grade, rather than not stratify for degeneration at all. For example, regarding Pfirrmann grade 2, it cannot be excluded that the lower T2-time in the lower lumbar spine reflects a natural higher, within-grade, degeneration process caudally as compared to cranially. Speaking against solely such an effect is the higher T2-time within the region anterior annulus-nucleus pulposus (ROI2) in IVDs in the lower lumbar spine compared to cranially. For example, the mean T2-time in ROI2 at L5/S1 was $9 \%$ higher as compared to ROI2 in L1/L2.

\section{Limitation}

Despite 606 included IVDs, there were no Pfirrmann grade 5 IVDs and few observations at several levels for Pfirrmann grade 3 and grade 4 IVDs, which limits generalization of the results in degenerated IVDs, especially in the absence of statistical significance. This needs to be addressed in future studies. Even though this exploratory study was not designed to test for agreement, and did not allow stratification for all lumbar levels for all Pfirrmann grades, the aim was to investigate whether T2-time level-dependent differences do exist when performing T2-mapping, which this study shows 
within both non-degenerated and degenerated IVDs. A large proportion of this cohort of healthy young adults was highly physically active [22], which does not reflect the general population. However, it is reasonable to assume that similar level-dependent differences exist also in a population with a wider age range and with a more moderate activity level.

\section{Conclusion}

Significant level-dependent T2-time differences within several Pfirrmann grades, both for the entire IVD volume and for multiple IVD subregions, show that the leveldependent influence on T2-time cannot be neglected. Even though between-level T2-time differences were most apparent within non-degenerated IVDs, they also existed within degenerated IVDs. Quantitative imaging techniques are emerging in order to improve non-invasive diagnostics within LBP. This work is therefore an important base for future research within the field, proving that it is necessary to stratify for lumbar level when quantitative IVD studies are performed using T2-mapping.

Acknowledgements Open access funding provided by University of Gothenburg. This project was supported by the School of Exercise and Nutrition Sciences, Deakin University (Grant ID: Belavy 2014-2017). The study was also financed by grants from the Swedish state under the agreement between the Swedish government and the county councils, the ALF agreement ALFGBG-792231. Gerontology Research Center is a joint effort between the University of Jyvaskyla and the University of Tampere.

\section{Compliance with ethical standards}

Conflict of interest All authors have declared that they have no conflicts of interest.

Ethical approval All participants gave their informed written consent prior to participation in the study. The study was performed in accordance with the ethical standards of the institutional and/or national research committee and with the 1964 Helsinki declaration and its later amendments or comparable ethical standards.

Open Access This article is distributed under the terms of the Creative Commons Attribution 4.0 International License (http://creativeco mmons.org/licenses/by/4.0/), which permits unrestricted use, distribution, and reproduction in any medium, provided you give appropriate credit to the original author(s) and the source, provide a link to the Creative Commons license, and indicate if changes were made.

\section{References}

1. Pfirrmann CW et al (2001) Magnetic resonance classification of lumbar intervertebral disc degeneration. Spine (Phila Pa 1976) 26(17):1873-1878
2. Nilsson $\mathrm{M}$ et al (2016) Axial loading during MRI influences T2-mapping values of lumbar discs: a feasibility study on patients with low back pain. Eur Spine J 25(9):2856-2863

3. Yamabe D et al (2017) Evaluation of water content in lumbar intervertebral discs and facet joints before and after physiological loading using T2 mapping MRI. Spine 42(24):E1423-E1428

4. Chokan $\mathrm{K}$ et al (2016) Evaluation of water retention in lumbar intervertebral disks before and after exercise stress with T2 mapping. Spine 41(7):E430-E436

5. Belavý DL et al (2017) Running exercise strengthens the intervertebral disc. Sci Rep 7:45975

6. Blumenkrantz $\mathrm{G}$ et al (2010) In vivo 3.0-tesla magnetic resonance T1rho and T2 relaxation mapping in subjects with intervertebral disc degeneration and clinical symptoms. Magn Reson Med 63(5):1193-1200

7. Takashima $\mathrm{H}$ et al (2012) Correlation between $\mathrm{T} 2$ relaxation time and intervertebral disk degeneration. Skelet Radiol 41(2):163-167

8. Welsch GH et al (2011) Parametric T2 and T2* mapping techniques to visualize intervertebral disc degeneration in patients with low back pain: initial results on the clinical use of 3.0 Tesla MRI. Skelet Radiol 40(5):543-551

9. Ogon I et al (2015) Analysis of chronic low back pain with magnetic resonance imaging T2 mapping of lumbar intervertebral disc. J Orthop Sci 20(2):295-301

10. Messner A et al (2017) Does T2 mapping of the posterior annulus fibrosus indicate the presence of lumbar intervertebral disc herniation? A 3.0 Tesla magnetic resonance study. Eur Spine J 26(3):877-883

11. Abdollah V (2017) The effects of axial loading on the disc and motion segments relative to disc degeneration and pain using novel MRI biomarkers, in Faculty of Rehabilitation Medicine. University of Alberta: https://era.library.ualberta.ca/files/cnz80 5z93w/Abdollah_Vahid_201701_PhD.pdf

12. Hebelka $\mathrm{H}$ et al (2018) Axial loading during MRI reveals deviant characteristics within posterior IVD regions between low back pain patients and controls. Eur Spine J 27:1-7

13. Stelzeneder D et al (2011) Quantitative in vivo MRI evaluation of lumbar facet joints and intervertebral discs using axial T2 mapping. Eur Radiol 21(11):2388-2395

14. Trattnig $\mathrm{S}$ et al (2010) Lumbar intervertebral disc abnormalities: comparison of quantitative T2 mapping with conventional MR at 3.0 T. Eur Radiol 20(11):2715-2722

15. Hoppe $\mathrm{S}$ et al (2012) Axial T2 mapping in intervertebral discs: a new technique for assessment of intervertebral disc degeneration. Eur Radiol 22(9):2013-2019

16. Mok GS et al (2016) Comparison of three approaches for defining nucleus pulposus and annulus fibrosus on sagittal magnetic resonance images of the lumbar spine. J Orthop Trans1 6:34-41

17. Antoniou J et al (2013) Analysis of quantitative magnetic resonance imaging and biomechanical parameters on human discs with different grades of degeneration. J Magn Reson Imaging 38(6):1402-1414

18. Zhu T et al (2015) Segmental quantitative MR imaging analysis of diurnal variation of water content in the lumbar intervertebral discs. Korean J Radiol 16(1):139-145

19. Pooni J et al (1986) Comparison of the structure of human intervertebral discs in the cervical, thoracic and lumbar regions of the spine. Surg Radiol Anat 8(3):175-182

20. Zirbel SA et al (2013) Intervertebral disc degeneration alters lumbar spine segmental stiffness in all modes of loading under a compressive follower load. Spine J 13(9):1134-1147

21. Abdollah V, Parent EC, Battié MC (2018) MRI evaluation of the effects of extension exercises on the disc fluid content and location of the centroid of the fluid distribution. Musculoskelet Sci Pract 33:67-70 
22. Belavy DL et al (2018) Specific modulation of vertebral marrow adipose tissue by physical activity. J Bone Miner Res 33(4):651-657

23. Benjamini Y, Hochberg Y (1995) Controlling the false discovery rate: a practical and powerful approach to multiple testing. J R Stat Soc Ser B (Methodol) 57(1):289-300

24. Alexander LA et al (2007) The response of the nucleus pulposus of the lumbar intervertebral discs to functionally loaded positions. Spine 32(14): 1508-1512

25. Stelzeneder D et al (2012) Quantitative T2 evaluation at 3.0T compared to morphological grading of the lumbar intervertebral disc: a standardized evaluation approach in patients with low back pain. Eur J Radiol 81(2):324-330

26. Watanabe A et al (2007) Classification of intervertebral disk degeneration with axial T2 mapping. Am J Roentgenol 189(4):936-942

27. Hebelka $\mathrm{H}$ et al (2018) Axial loading during MRI induces significant T2 value changes in vertebral endplates-a feasibility study on patients with low back pain. J Orthop Surg Res 13(1):18

Publisher's Note Springer Nature remains neutral with regard to jurisdictional claims in published maps and institutional affiliations.

\title{
Affiliations
}

\author{
H. Hebelka ${ }^{1,4}$ (1) K. Lagerstrand ${ }^{3,4} \cdot$ H. Brisby ${ }^{2,4} \cdot$ P. J. Owen ${ }^{5} \cdot$ M. J. Quittner ${ }^{5}$ T. Rantalainen ${ }^{5,6} \cdot$ D. L. Belavy ${ }^{5}$ \\ H. Hebelka \\ hanna.hebelka@vgregion.se \\ 1 Department of Radiology, Sahlgrenska University Hospital, \\ Gothenburg, Sweden \\ 2 Department of Orthopaedics, Sahlgrenska University \\ Hospital, Gothenburg, Sweden \\ 3 Department of Medical Physics and Biomedicine, \\ 4 Institute of Clinical Sciences, Sahlgrenska Academy, \\ University of Gothenburg, Gothenburg, Sweden \\ 5 Institute for Physical Activity and Nutrition, School \\ of Exercise and Nutrition Sciences, Deakin University, \\ Geelong, VIC, Australia \\ 6 Gerontology Research Center and Faculty of Sport \\ and Health Sciences, University of Jyväskylä, Jyvaskyla, \\ Finland
} Sahlgrenska University Hospital, Gothenburg, Sweden 